\title{
Egg quality and fatty acid profile of yolk in eggs from Athulya, native and IWN x native crossbred layers under backyard system"
}

\author{
S. Devavratha', Binoj Chacko ${ }^{2}$, P. Anitha ${ }^{3}$, S. Harikrishnan ${ }^{4}$ and V. Ramnath ${ }^{5}$ \\ Department of Poultry Science, \\ College of Veterinary and Animal Sciences, Mannuthy, Thrissur, Kerala 680651 \\ Kerala Veterinary and Animal Sciences University, India.
}

Citation: Devavratha, S., Binoj Chacko, Anitha, P., Harikrishnan, S. and Ramnath, V. 2021. Egg quality and fatty acid profile of yolk in eggs from Athulya, native and IWN $\mathrm{x}$ native crossbred layers under backyard system. J. Vet. Anim. Sci. 52(4): 362-366

DOI: https://doi.org/10.51966/jvas.2021.52.4.362-366

Received:20.02.2021

Accepted: 19.03.2021

Published:15.12.2021

\section{Abstract}

Backyard poultry production forms the basis for transforming the rural poultry sector from subsistence to a more economically productive base. The current study was conducted to evaluate the egg quality of eggs produced by Athulya, native and IWN $x$ Native crossbred layers under backyard system. At 36 weeks of age, one egg from each group of birds was collected for fatty acid estimation of egg yolk and 20 eggs from each group was collected at 40 weeks of age for assessing the haugh unit score, yolk colour, shell thickness and shell colour. The beneficial polyunsaturated fatty acid content was higher in native bird eggs. The eggs from Athulya and native chicken had better internal quality in terms of haugh unit and yolk colour score. The shell thickness of eggs was similar among three groups. The egg shell colour was white in Athulya, tinted to brown in native and tinted in IWN $x$ Native crossbreds and the shell colour in native and crossbreds exhibits more consumer preference.

Keywords: Backyard, fatty acid profile, egg quality

Running title: Egg quality and fatty acid profile of yolk

Rural backyard poultry provides nutritional supplements in the form of valuable nutrients through eggs. In a free-range chicken system, grass, herbs, insects that live in soil and plants

"Part of MVSc thesis submitted to Kerala Veterinary and Animal Sciences University, Pookode, Wayanad, Kerala

1. MVSc Scholar and *Corresponding author:email-mplyprasad@gmail.com,Ph: 9744020837

2. Assistant Professor and Head, University Poultry and Duck Farm

3. Professor and Head

4. Assistant Professor

5. Professor and Head, Department of Veterinary Physiology

Copyright: @ 2021 Devavratha et al. This is an open access article distributed under the terms of the Creative Commons Attribution 4.0 International License (http://creativecommons.org/licenses/by/4.0/), which permits unrestricted use, distribution, and reproduction in any medium, provided the original author and source are credited. 
enrich chicken food and they may assimilate natural nutrients in their eggs. The internal and external quality of the egg is an important trait in both intensive and extensive system of poultry production. The egg quality can be affected by many factors such as breed, feed, temperature, storage, rearing conditions, diseases etc Eggs with higher haugh unit score possess better internal quality. The darker yolk coloured eggs has more consumer preference and this colour of yolk is affected mainly by xanthophyll content of the feed. Eggs with optimum shell thickness indicates a better external quality of the egg and egg shell colour is an important trait that influence the consumer acceptability and marketability of eggs. The consumption of unsaturated fatty acids is associated with the reduction of undesirable cholesterol and $\omega-3$ polyunsaturated fatty acids are relevant nutrients in egg yolks and are considered as essential fatty acids. The fatty acid content, internal and external quality of eggs can vary in different breeds of chicken when reared under backyard system. The objective of the present study is to evaluate the egg quality and fatty acid profile of yolk in eggs from Athulya, native and IWN x Native birds reared under backyard system.

\section{Materials and methods}

\section{Experimental layout}

The study was conducted using three group of layer birds namely, Athulya, native and IWN x Native under backyard system of rearing. The experiment birds were reared under deep litter system up to 14 weeks of age. Thirty households were selected and a total of fifty birds were distributed from each group so that each household received a set of five birds. At 36 weeks of age, one egg from each group was randomly selected and a total of three eggs was utilised for fatty acid estimation of yolk. The collected eggs as samples for fatty acid estimation was analysed at Animal Feed Analytical and Quality Assurance Laboratory (AFAQAL), Namakkal, TamilNadu by gas chromatography method. At 40 weeks of age, 20 eggs from each group was collected and was utilised to assess the haugh unit score, yolk colour score, shell thickness and shell colour.

\section{Estimation of egg quality and fatty acid content of egg yolk}

Two eggs from each household were randomly collected and a total of 20 eggs were subjected to measure the internal qualities of egg. The eggs were carefully broken without damaging the albumen and yolk for studying the internal qualities. The height of albumen was measured using Ame's tripod stand micro meter. The yolk colour score was assessed using DSM yolk colour fan. Shell thickness was measured using screw gauge micro meter and expressed in $\mathrm{mm}$. Haugh unit score was calculated from height of albumen and weight of respective eggs.

The formula for calculating the Haugh unit score is given below

Haughunit=100xlog $\left(\mathrm{H}-1.7 \mathrm{~W}^{0.37}+7.6\right)$, where $\mathrm{H}$ stands for height of albumen $(\mathrm{mm})$ and W stands for weight of eggs $(\mathrm{g})$.

One egg was randomly selected from each treatment group at 36 weeks of age and the sample egg were subjected to fatty acid profile estimation using Gas chromatography as per O'Fallon et al. (2007). Results were expressed as percentage. The difference in relative content of saturated and unsaturated fatty acids of the eggs was recorded.

\section{Statistical analysis}

Data collected on egg quality parameters during the experiment were statistically analyzed by means of one-way ANOVA using SPSS Version 24.0. The statistical analysis on fatty acid profile of egg yolk was not done as the data was obtained from pooled samples.

\section{Results and discussion}

\section{Fatty acid content of egg samples}

The fatty acid content of Athulya, native and IWN $x$ Native crossbred eggs at 36 weeks of age is listed in the table 1.

Fatty acid content of egg yolk is affected by the hen's diet. Higher inclusion 
Table 1. Fatty acid profile of Athulya, native and IWN x Native crossbred eggs at 36 weeks of age

\begin{tabular}{|l|l|l|c|}
\hline \multirow{2}{*}{ Parameters } & \multicolumn{3}{|c|}{ Treatment groups } \\
\cline { 2 - 4 } & Athulya & Native & IWNx Native \\
\hline Myristic acid (\%) & 2.08 & 1.77 & 1.10 \\
\hline Palmitic acid (\%) & 29.98 & 29.58 & 32.65 \\
\hline Stearic acid (\%) & 11.08 & 9.94 & 8.72 \\
\hline Behenic acid (\%) & 4.03 & 4.74 & 3.13 \\
\hline Oleic acid (\%) & 24.33 & 26.38 & 34.85 \\
\hline Palmitoleic acid (\%) & 5.76 & 5.96 & 3.57 \\
\hline Arachidonic acid (\%) & 0.08 & 0.03 & 0.08 \\
\hline Linoleic acid (\%) & 9.11 & 11.89 & 7.69 \\
\hline Eicosapenteanoic acid (\%) & 5.86 & 6.22 & 4.79 \\
\hline Docosahexaenoic acid (\%) & 3.78 & 3.34 & 3.21 \\
\hline Linolenic acid (\%) & 0.12 & 0.09 & 0.17 \\
\hline$\omega-6$ (\%) & 9.19 & 11.92 & 7.77 \\
\hline$\omega-3$ (\%) & 9.76 & 9.65 & 8.17 \\
\hline$\omega-6: \omega-3$ & 1.06 & 0.809 & 1.05 \\
\hline Total SFA (\%) & 47.17 & 46.03 & 45.60 \\
\hline Total MUFA (\%) & 30.09 & 32.24 & 38.42 \\
\hline Total PUFA (\%) & 18.95 & 21.57 & 15.94 \\
\hline
\end{tabular}

Table 2. Egg quality parameters of eggs from Athulya, native and IWN x Native crossbred layers at 40 weeks of age are listed below

\begin{tabular}{|c|c|c|c|c|}
\hline Group & $\begin{array}{c}\text { Haugh Unit } \\
\text { score }\end{array}$ & $\begin{array}{c}\text { Yolk colour } \\
\text { score }\end{array}$ & $\begin{array}{c}\text { Shell thickness } \\
(\mathbf{m m})\end{array}$ & Shell colour \\
\hline Athulya & $75.33^{\mathrm{b}} \pm 2.47$ & $8.37^{\mathrm{b}} \pm 0.33$ & $0.35 \pm 0.02$ & White \\
\hline Native & $73.18^{\mathrm{b}} \pm 2.98$ & $7.79^{\mathrm{b}} \pm 0.26$ & $0.33 \pm 0.01$ & Tinted to brown \\
\hline IWN x Native & $64.41^{\mathrm{a}} \pm 2.60$ & $6.74^{\mathrm{a}} \pm 0.30$ & $0.33 \pm 0.00$ & Tinted \\
\hline
\end{tabular}

Mean values bearing different superscripts differed significantly within a column $(p<0.05)$

of monounsaturated fatty acids and polyunsaturated fatty acid than saturated fatty acid in human diet can reduce cholesterol level. In the present study, the fatty acid contents in the eggs of all the three groups did not differ much. The PUFA content was higher in eggs of native birds. The saturated fatty acid value was lower in IWN x Native birds. The MUFA value was higher in IWN $x$ Native crossbreds. The difference in fatty acid content of eggs might be due to breed effect.

In agreement with the present findings, Sarma et al. (2018) reported that the most favourable PUFA ratio was higher in Desi chicken eggs of Assam. Contrary to this, Arsha (2019) recorded a higher SFA and PUFA content in eggs of Athulya (48.30 and 15.65) than native birds (46.37 and 13.04) and a lower
MUFA value in Athulya (33.78 and 4.61) than native birds (38.89 and 5.15) under intensive system of rearing. Cherian etal. (2002) recorded a similar palmitoleic acid content as that of IWN $x$ Native bird. From the nutritional point of view, the $\omega 6: \omega 3$ ratio is better in eggs from free range (Simcic et al., 2011). The lower $\omega 6$ : $\omega 3$ ratio in Desi chicken eggs indicated its superiority over other eggs in reducing cardiovascular risks (Sarma et al., 2018).

\section{Egg quality traits}

The Haugh unit score of an egg is a measure of albumen quality and a higher Haugh unit score indicates a better internal egg quality. There are many factors such as age, breed or strain of hen, dietary ingredients, storage conditions and possible diseases, which affect 
haugh unit values(Roberts, 2004). In the present study, it was found that the birds from IWN $x$ Native crossbreds (64.41) produced eggs with lower albumen height and haugh unit values at 40 weeks of age indicating poor internal egg quality compared to eggs from Athulya and native birds. The lower internal egg quality in IWN x Native crossbreds is in agreement with reports of Harikrishnan et al. (2019) in the same crossbred. The present findings of difference in internal egg quality of the three groups can be attributed to the genotypic differences. The haugh unit score of eggs of Athulya and native birds was almost similar in the present study which is in contrary to findings of Lordelo et al. (2017) who reported a lower haugh unit score in eggs of commercial hybrids than native Portuguese breeds.

A darker yolk colour is preferred by egg consumers. The colour of egg yolk is influenced by factors like xanthophyll in the feed (Whiting et al., 2019), breed or strain of bird and possible diseases affecting pigment or fat metabolism. In the present study, eggs from birds in Athulya and native group had darker yolk colour. The yolk colour was lighter in eggs laid by IWN $x$ Native crossbreds which was in accordance with the reports of Harikrishnan et al. (2019) in the same crossbred. The genotypic influence on yolk colour was also reported by Lordelo et al. (2017) in which yolk colour in the eggs of native chicken was lighter than commercial hybrids. In the present study, birds from all the three groups had access to green under backyard rearing which was the reason for darker egg yolk colour compared to previously reported results for the same group of birds in intensive system (AICRP, 2016).

There are many factors such as age, breed or strain of bird, feed, ambient temperature and diseases that affect shell thickness (Sreenivas et al., 2013). Eggs with shell thickness $\leq 0.30 \mathrm{~mm}$ are considered as thin shelled eggs. In the present study, the egg shell thickness in all the three groups were similar and optimum. This indicates that the feed resources available in the households were sufficient to meet the calcium requirements of these birds for proper egg shell formation. The egg shell thickness in the present study was higher than previously published data for the same breeds in intensive system by AICRP (2016) but lower than egg shell thickness reported by Harikrishnan (2019) which might be due to variation in feeding, housing and ambient temperature.

Even though egg shell colour is not an indicator of nutritive value, it is an important factor influencing consumer preference (Cavero et al., 2008). Generally, egg shell colour is a breed characteristic but the intensity of the shell colour can vary within the breed or strain. In the present study, the egg shell colour was white in Athulya, tinted to brown in native and tinted in crossbred birds. The difference in egg shell colour observed among the groups in the present study was due to different genotype of the birds.

\section{Conclusion}

Current findings showed that eggs of native chicken contained more beneficial fatty acids. Athulya and native chicken eggs under backyard rearing showed better internal quality than IWN $x$ native chicken eggs. The shell thickness was optimum for three groups of birds and the shell colour was favourable for native and IWN $x$ native birds regarding consumer preference.

\section{Acknowledgement}

The authors are thankful to the Kerala Veterinary and Animal Sciences University for providing the facilities needed for carrying out the research.

\section{Conflicts of interest}

There were no conflicts of interest reported by the authors.

\section{References}

AICRP [All India Co-ordinated Research Project]. 2016. Annual report 2015-16. All India Co-ordinated Research Project on Poultry Improvement for Eggs, Mannuthy Centre, 32p. 
Arsha. V. S. 2019. Influence of Fish Oil on Production Performance and Fatty Acid Profile of Eggs in Athulya and Native Chicken. M.V.Sc.Thesis, Kerala Veterinary and Animal Sciences University, Pookode, 107p.

Cavero, D., Icken, W., Thurner, S., Schmutz, M., Wendl, G. and Preisinger, R., 2008. Relationship between time spent in the winter garden and shell colour in brown egg stock. Arch. Für Geflügelkd. 75: 145-150.

Cherian, G., Holsonbake, T. B. and Goeger, M. P. 2002. Fatty acid composition and egg components of specialty eggs. Poult. Sci. 81: 30-33.

Harikrishnan, S., Binoj Chacko, P. Anitha, Beena C. Joseph, K. Anilkumar and Joseph Mathew. 2019. Performance of the Production Characteristics of the Reciprocal Crosses of Native Chicken of Kerala and N Strain (IWN) of White Leghorn. Int. J. Curr. Microbiol. App. Sci. 8: $1562-1567$.

Lordelo, M., Fernandes, E., Bessa, R. J. B. and Alves, S. P., 2017. Quality of eggs from different laying hen production systems, from indigenous breeds and specialty eggs. Poult. Sci. 96: 1485-1491.
O' Fallen, J.V., Bushboom, J. R., Nelson, M. L. and Gaskins, C.T.2007. A direct method for fatty acid methyl ester sysnthesis: application to wet meat tissues, oils, and feed stuffs. J. Anim. Sci. 85: 1511- 1521.

Roberts, J. R., 2004. Factors affecting egg internal quality and egg shell quality in laying hens. J. Poult. Sci. 41: 161-177.

Sarma, M., Islam, R., Borah, M. K., Sharma, P., Mahanta, J. D., Kalita, N. and Bhattacharyya, B. N. 2018. Comparative performance of Vanaraja, Srinidhi and Desi chicken under traditional system among tribal community of Assam. Indian J. Anim. Res. 52: 1518-1520.

Simcic, M., Stibilj, V. and Holcman, A. 2011. Fatty acid composition of eggs produced by the Slovenian Autochthonous Styrian hen. Food Chem. 3: 873-877.

Sreenivas, D., Prakash, M.G., Mahender, M. and Chatterjee, R.N., 2013. Genetic analysis of egg quality traits in White Leghorn chicken. Vet. WId. 6: 260-263.

Whiting, I.M., Rose, S.P., Mackenzie, A.M., Amerah, A.M. and Pirgozliev, V.R. 2019. Effect of wheat distillers dried grains with solubles and exogenous xylanase on laying hen performance and egg quality. Poult. Sci. 9: 3756-3762. 